\title{
SAND2000-1304C
}

\section{Mechanisms Affecting Emission in Rare-Earth-Activated Phosphors}

\author{
David R. Tallant, Carleton H. Seager and Regina L. Simpson \\ Sandia National Laboratories, Albuquerque, NM, 87185-1411, U.S.A.
}

\begin{abstract}
The relatively poor efficiency of phosphor materials in cathodoluminescence with low accelerating voltages is a major concern in the design of field emission flat panel displays operated below $5 \mathrm{kV}$. Our research on rare-earth-activated phosphors indicates that mechanisms involving interactions of excited activators have a significant impact on phosphor efficiency. Persistence measurements in photoluminescence (PL) and cathodoluminescence (CL) show significant deviations from the sequential relaxation model. This model assumes that higher excited manifolds in an activator de-excite primarily by phonon-mediated sequential relaxation to lower energy manifolds in the same activator ion. In addition to sequential relaxation, there appears to be strong coupling between activators, which results in energy transfer interactions. Some of these interactions negatively impact phosphor efficiency by nonradiatively de-exciting activators. Increasing activator concentration enhances these interactions. The net effect is a significant degradation in phosphor efficiency at useful activator concentrations, which is exaggerated when low-energy electron beams are used to excite the emission.
\end{abstract}

\section{INTRODUCTION}

Phosphors are the light-emitting components used in flat panel displays based on electroluminescent, plasma or field emission excitation technologies. Unlike cathode ray tube (CRT) displays, which use highly energetic electrons and substantial beam currents to excite phosphor emission, design constraints in flat panel displays severely limit the amount of excitation power available to excite phosphors. Consequently, the emission efficiency of phosphor materials becomes an issue of major concern in the design of flat panel displays. Unfortunately, the emission efficiency of rare-earth-activated phosphors appears to be limited by concentration quenching of activators (the dopants which produce characteristic light emission in phosphors) [1] and other effects [2]. The quenching effects are aggravated when low-voltage electrons are used for excitation [3]. It is, therefore, important to understand the mechanisms of quenching in phosphors and so determine whether quenching limitations of phosphor efficiency can be overcome and, if so, how. Our work has identified interactions between activators in excited states which have a major impact on phosphor efficiency. This paper presents evidence for these interactions (primarily) from photoluminescence (PL) experiments but also with data from cathodoluminescence (CL) which show concomitant effects.

\section{EXPERIMENTAL DETAILS}

Powder samples of phosphors from the systems $\mathrm{Y}_{2} \mathrm{O}_{3}: \mathrm{Eu}, \mathrm{Y}_{2} \mathrm{SiO}_{5}: \mathrm{Tb}$ and $\mathrm{Zn}_{2} \mathrm{SiO}_{4}: \mathrm{Mn}$ were prepared by spray pyrolysis (Superior MicroPowders) in dopant concentrations from 0.2 to 10 atomic \%. Photoluminescence (PL) emission spectra were obtained using a $\mathrm{D}_{2}$ lamp/monochromator combination for excitation and a $0.6-\mathrm{m}$ spectrograph with a chargecoupled device detector to disperse and record the emitted light. PL persistence (light intensity versus time) curves were obtained using a Nd:YLF pump laser and optical parametric oscillator to provide pulsed, ultraviolet and visible excitation and a $0.6-\mathrm{m}$ spectrograph, photomultiplier 


\section{DISCLAIMER}

This report was prepared as an account of work sponsored by an agency of the United States Government. Neither the United States Government nor any agency thereof, nor any of their employees, make any warranty, express or implied, or assumes any legal liability or responsibility for the accuracy, completeness, or usefulness of any information, apparatus, product, or process disclosed, or represents that its use would not infringe privately owned rights. Reference herein to any specific commercial product, process, or service by trade name, trademark, manufacturer, or otherwise does not necessarily constitute or imply its endorsement, recommendation, or favoring by the United States Government or any agency thereof. The views and opinions of authors expressed herein do not necessarily state or reflect those of the United States Government or any agency thereof. 


\section{DISCLAIMER}

Portions of this document may be illegible in electronic image products. Images are produced from the best available original document. 
tube and digital storage oscilloscope to disperse, detect and capture light intensity transients. Cathodoluminescence (CL) persistence measurements were obtained at accelerating voltages from 0.8 to $4 \mathrm{keV}$. Emission spectra were recorded using a small spectrograph and a chargecoupled device detector. Light transients were captured using wavelength-isolating optical filters, a photomultiplier tube and a digital storage oscilloscope.

\section{RESULTS AND DISCUSSION}

A primary limitation to increasing phosphor efficiencies is that the individual activators in essentially all phosphor systems become less efficient at converting input energy to light output as the activator concentration is increased. As shown in Figure 1, the output intensity of (red) light from the $\mathrm{Eu}{ }^{5} \mathrm{D}_{0}$ energy level divided by the Eu activator concentration (a value proportional to the efficiency per Eu activator) decreases tenfold over the 0.2 to 9 atomic \% range of Eu concentration for PL excitation at $260 \mathrm{~nm}$. CL efficiency per activator decreases even more over the same activator concentration range for an accelerating voltage of $3 \mathrm{keV}$ and degrades further if lower accelerating voltages are used. Both PL and CL efficiency per activator values were normalized to 1.0 for the $\mathrm{Y}_{2} \mathrm{O}_{3}: 0.2$ atomic \% Eu powder

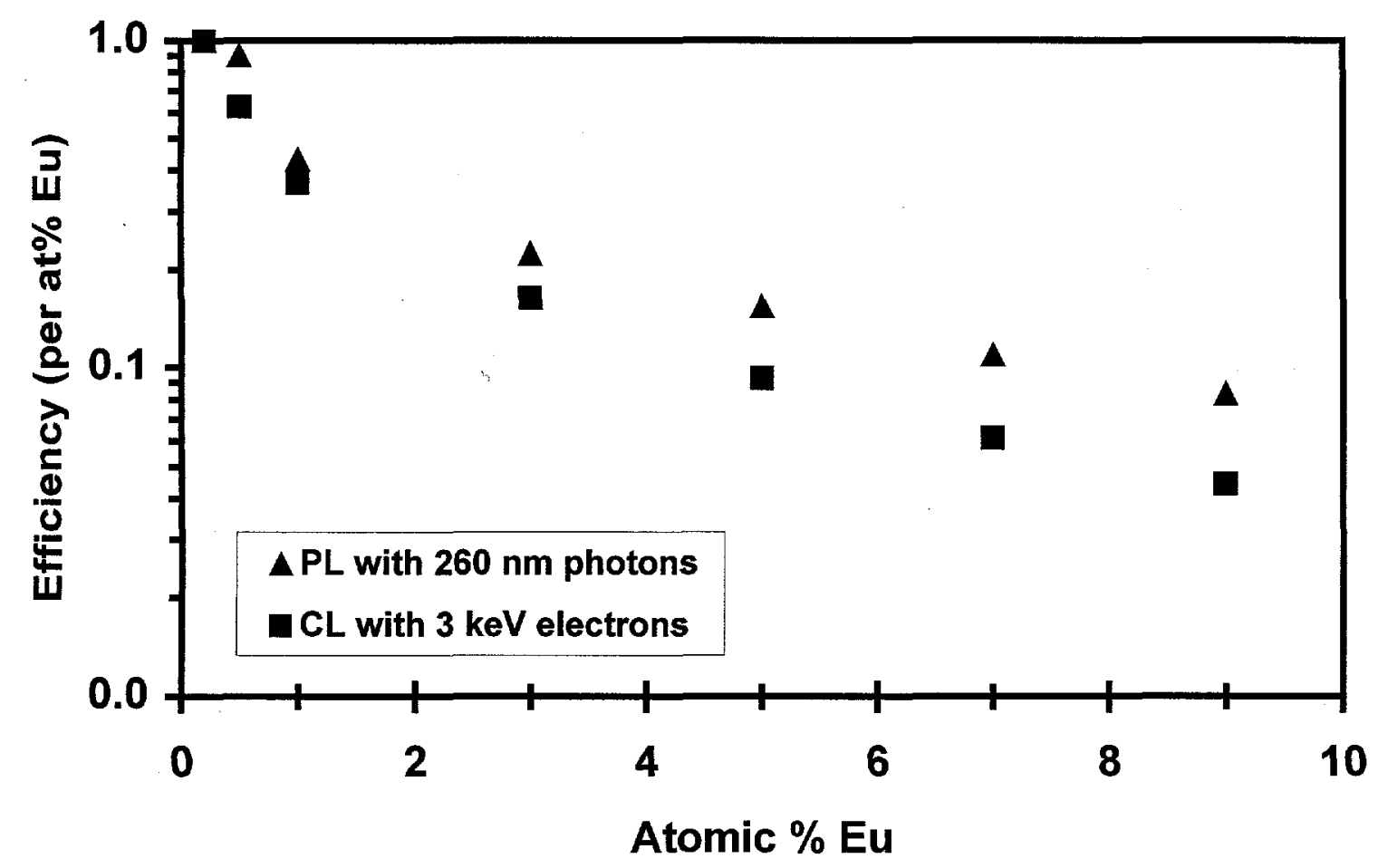

Figure 1. Normalized $\mathrm{Y}_{2} \mathrm{O}_{3}: \mathrm{Eu}^{5} \mathrm{D}_{0}$ (Red) emission per activator for PL excited at $260 \mathrm{~nm}$ and $\mathrm{CL}$ excited with $3 \mathrm{keV}$ electrons.

The decrease in efficiency per activator shown in Figure 1 as a function of activator concentration implies that the rate of a process which de-excites the activators nonradiatively, i.e., without photon emission, is increasing with increasing activator concentration. This increase 
in the nonradiative de-excitation rate increases the overall rate of depopulation of the excited states. Stated in other terms, the persistence lifetimes of the excited states of the Eu activators should decrease as the activator concentration increases, due to an increased rate of depopulation from nonradiative de-excitation events.

The energy level diagram for $\mathrm{Eu}^{3+}$ is shown in Figure 2. $\mathrm{Eu}^{3+}$ has a ground state of ${ }^{7} \mathrm{~F}$ manifolds. The lowest excited states are of ${ }^{5} \mathrm{D}$ character, with the ${ }^{5} \mathrm{D}_{0}$ state having the longest lifetime and emitting the red light characteristic of $\mathrm{Eu}^{3+}$-activated phosphors. Above the ${ }^{5} \mathrm{D}$ states are closely spaced states of $\mathrm{f}$-orbital character and then charge transfer bands [3]. When a deepultraviolet $(250 \mathrm{~nm})$ photon is absorbed by $\mathrm{Eu}^{3+}$, the $\mathrm{Eu}^{3+}$ is excited into a charge transfer band. For isolated $\mathrm{Eu}^{3+}$ ions (those incapable of transferring energy to another species in the phosphor lattice), the excitation relaxes sequentially through the various energy states of the ion, quickly to the ${ }^{5} \mathrm{D}_{3}$ level, then more slowly, but still sequentially through the ${ }^{5} \mathrm{D}$ levels to the ${ }^{5} \mathrm{D}_{0}$ level, which has a high probability of emitting a photon.

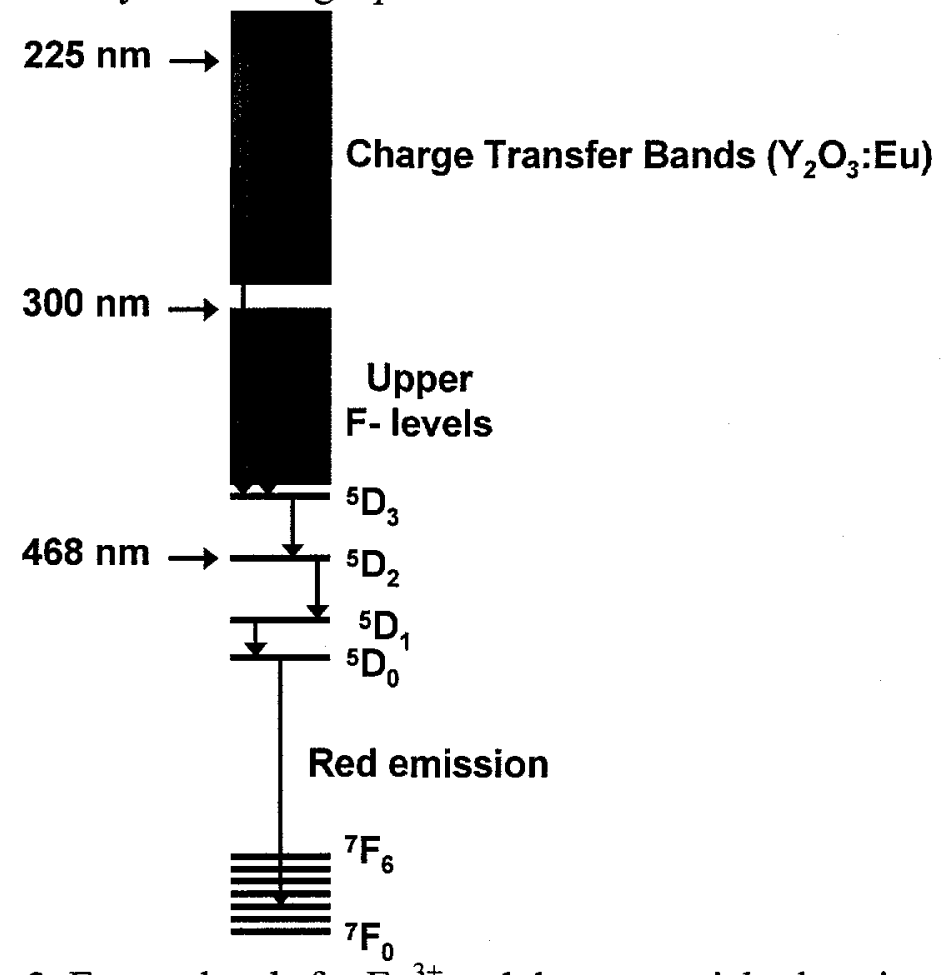

Figure 2. Energy levels for $\mathrm{Eu}^{3+}$ and the sequential relaxation model.

By exciting $\mathrm{Eu}^{3+}$ activators with ultraviolet photons at $225 \mathrm{~nm}$ from a pulsed source, we can excite detectable emission from the ${ }^{5} \mathrm{D}$ levels and obtain persistence curves from each of these levels. Relaxation within a single, isolated activator obeys first-order kinetics, and the persistence (emission intensity versus time) curve has an exponential, exp[-kt], dependence on time, $\mathrm{t}$, and the total relaxation rate, $\mathrm{k}\left(\mathrm{S}^{-1}\right)$. A semi-log plot of persistence is linear with slope, $\mathrm{k}$; whose inverse is the lifetime of the state whose emission is plotted. PL persistence curves were thus obtained for the $\mathrm{Y}_{2} \mathrm{O}_{3}$ :Eu powders whose emission efficiencies are shown in Figure 1. PL persistence lifetimes from the ${ }^{5} \mathrm{D}_{0},{ }^{5} \mathrm{D}_{1}$ and ${ }^{5} \mathrm{D}_{2}$ states for these powders are shown in Figure 3. 


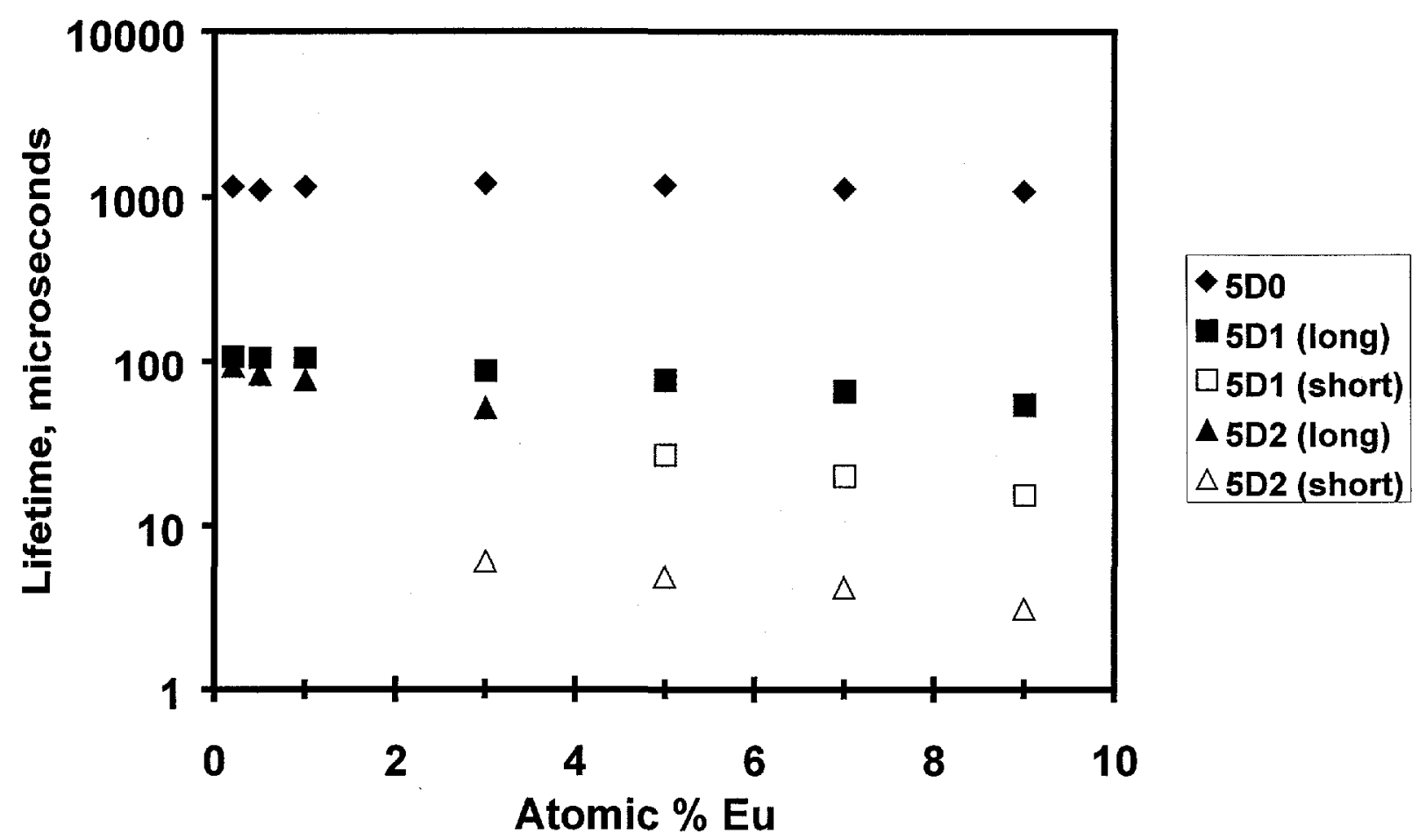

Figure 3. PL Persistence lifetimes for $\mathrm{Y}_{2} \mathrm{O}_{3}$ :Eu with $225 \mathrm{~nm}$ excitation.

The semi-log persistence plots for ${ }^{5} \mathrm{D}_{0}$ state emission are linear, and the lifetimes derived from these plots are constant over the range of activator concentrations shown in Figure 3. The emission efficiency of this state, which provides nearly all of the characteristic Eu ${ }^{3+}$ light output, must also be constant. The implications of this result are twofold. First, loss of activator efficiency as a consequence of concentration quenching in $\mathrm{Y}_{2} \mathrm{O}_{3}$ :Eu must be due to nonradiative processes operating on states above ${ }^{5} \mathrm{D}_{0}$. In other words, concentration quenching of $\mathrm{Y}_{2} \mathrm{O}_{3}:$ Eu emission is not due to loss of efficiency of the ${ }^{5} \mathrm{D}_{0}$ state, but it must be related to how excitation is transferred to the ${ }^{5} \mathrm{D}_{0}$ state. Second, this result is not consistent with quenching related to resonant or near-resonant energy transfer $[4,5,6]$ between activators. In this scenario excitation migration occurs until the activator emits light or the excitation reaches an activator near a "trap" site, which nonradiatively de-excites the activator. By this mechanism, quenching by trapping should most strongly affect ${ }^{5} \mathrm{D}_{0}$ state, since excitation will migrate the furthest in this, the longest-lived, excited $\mathrm{Eu}^{3+}$ state. Quenching by trapping will increase as increasing $\mathrm{Eu}^{3+}$ concentration enhances migration rates by reducing the average separation between $\mathrm{Eu}^{3+}$ ions. We conclude that such trapping effects (involving single, excited activators) are not strongly affecting the emission efficiency of $\mathrm{Y}_{2} \mathrm{O}_{3}: \mathrm{Eu}$.

The semi-log plots of persistence from the ${ }^{5} \mathrm{D}_{1}$ and ${ }^{5} \mathrm{D}_{2}$ states are linear at low $\mathrm{Eu}^{3+}$ concentrations, but, as the $\mathrm{Eu}^{3+}$ concentration reaches 3 atomic \%, they become nonlinear, indicating the advent of additional, non-first-order processes,. These nonlinear semi-log plots can be fit by equations involving more than one persistence lifetime. In Figure 3 these multiple lifetimes are plotted as long and short lifetime components of the ${ }^{5} \mathrm{D}_{1}$ and ${ }^{5} \mathrm{D}_{2}$ states. Both the 
long and short lifetime components decrease regularly with increasing $\mathrm{Eu}^{3+}$ concentration. The nonlinear appearance of the semi-log persistence plots from ${ }^{5} \mathrm{D}_{1}$ and ${ }^{5} \mathrm{D}_{2}$ emission and the decrease in ${ }^{5} \mathrm{D}_{1}$ and ${ }^{5} \mathrm{D}_{2}$ lifetimes with $\mathrm{Eu}^{3+}$ concentration suggest that non-first-order processes, which enhance the relaxation rates of these states, become increasingly important as the $\mathrm{Eu}^{3+}$ concentration increases.

We have carried out the same type of experiment for the $\mathrm{Y}_{2} \mathrm{SiO}_{5}: \mathrm{Tb}$ system and have obtained similar results. The $\mathrm{Tb}^{3+5} \mathrm{D}_{4}$ state, which is the longest-lived and provides nearly all the characteristic $\mathrm{Y}_{2} \mathrm{SiO}_{5}: \mathrm{Tb}$ (green) emission, has linear semi-log persistence plots and shows little variation in its lifetime over the 0.2 to 10 atomic $\%$ range of $\mathrm{Tb}^{3+}$ concentrations. The (higher in energy) ${ }^{5} \mathrm{D}_{3}$ state has nonlinear semi-log persistence plots over nearly this entire $\mathrm{Tb}^{3+}$ concentration range, and its lifetimes decrease by two-to-three orders of magnitude by 10 atomic $\% \mathrm{~Tb}^{3+}$.

The persistence plots in Figure 4 suggest the nature of the additional relaxation processes which are affecting the relaxation rates of the higher excited states in these phosphor systems. The figure includes the PL persistence curve obtained by exciting $\mathrm{Y}_{2} \mathrm{O}_{3}: 1$ atomic \% Eu with 225 $\mathrm{nm}$ excitation and monitoring ${ }^{5} \mathrm{D}_{0}$ emission $(611 \mathrm{~nm})$. Figure 4 also includes calculations from model [7] based on sequential relaxation (see Figure 2) and the ${ }^{5} \mathrm{D}_{0},{ }^{5} \mathrm{D}_{1}$ and ${ }^{5} \mathrm{D}_{2}$ lifetimes from Figure 3 . The lifetimes of the states above ${ }^{5} \mathrm{D}_{2}$ are too short to significantly affect the shape of the persistence curve for the time scale depicted. The long tail of the data and the calculated curve, which represents the lifetime of the ${ }^{5} \mathrm{D}_{0}$ state, match well, but the rise of the data is much faster than that of the calculated curve. The corresponding persistence curve from CL excitation likewise matches the ${ }^{5} \mathrm{D}_{0}$ lifetime, but it rises even faster (deviates even more from the calculated curve) than the PL persistence curve. The fast rises of the PL and CL curves must be due to a process which provides excitation to the ${ }^{5} \mathrm{D}_{0}$ state without passing through one or both of the intermediate states $\left({ }^{5} \mathrm{D}_{1}\right.$ and $\left.{ }^{5} \mathrm{D}_{2}\right)$. Figure 5 depicts a process by which this might occur.

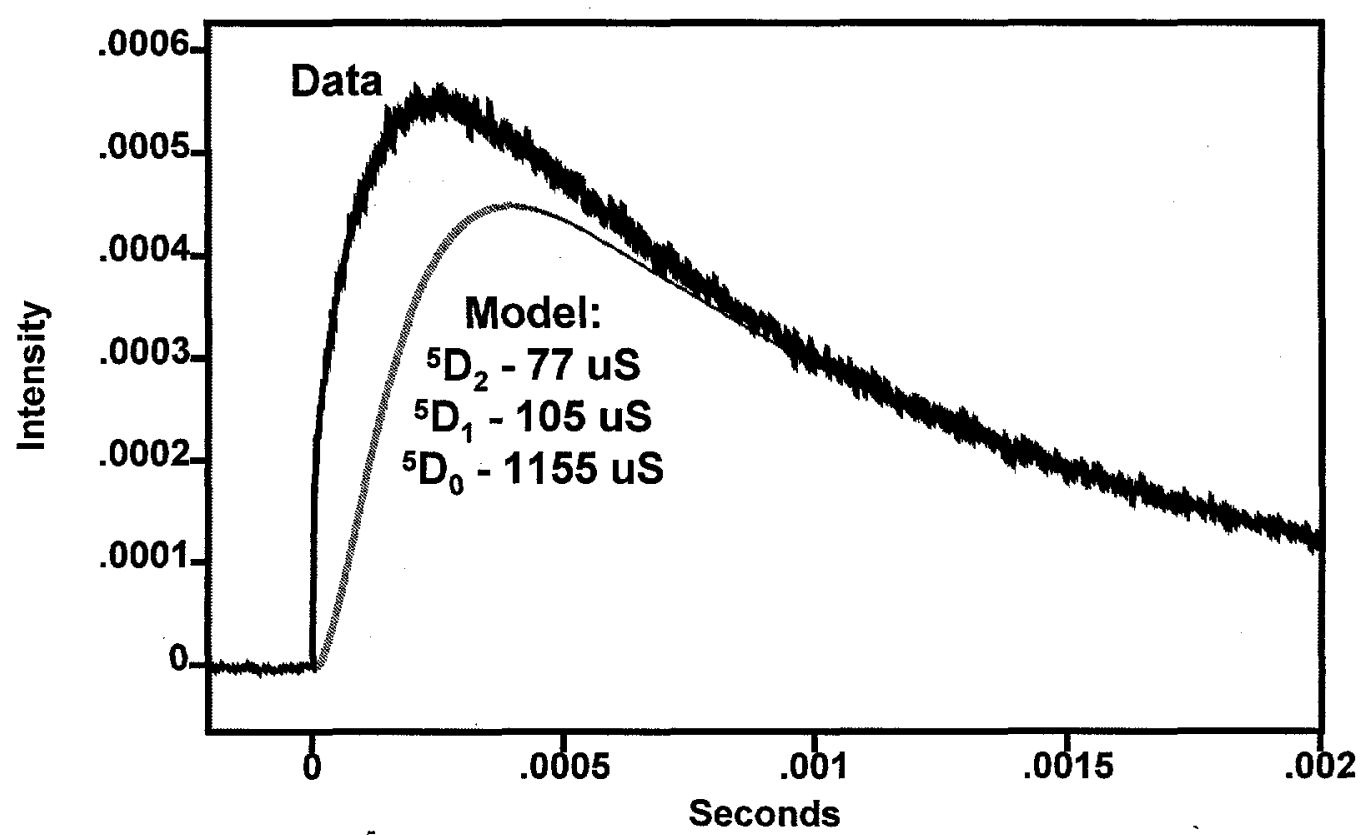

Figure 4. Persistence of ${ }^{5} \mathrm{D}_{0}$ emission ( $225 \mathrm{~nm}$ excitation) from $\mathrm{Y}_{2} \mathrm{O}_{3}$ : 1atomic $\% \mathrm{Eu}$ and curve calculated assuming sequential relaxation through excited states. 


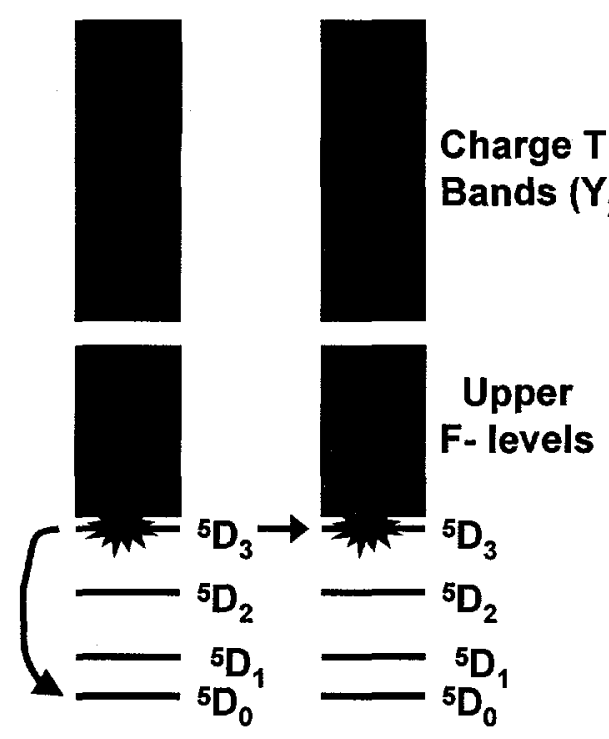

Two Activators in Excited States

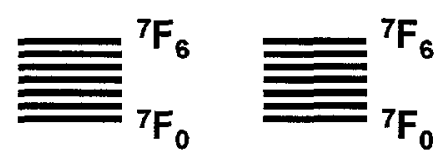

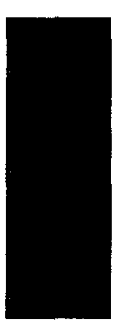
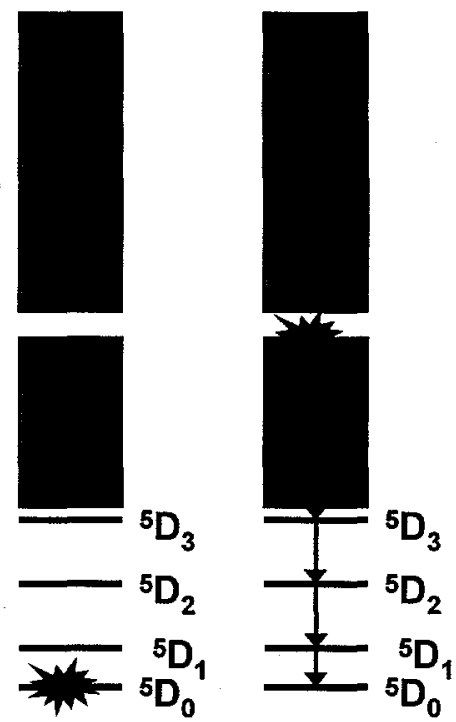

Two Potential Red Photons

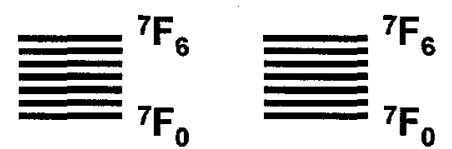

$7 F_{0}$

Figure 5. Direct Pumping of Eu ${ }^{5} \mathrm{D}_{0}$ by Excited State Energy Transfer

In Figure 5 two $\mathrm{Eu}^{3+}$ ions excited to the ${ }^{5} \mathrm{D}_{3}$ state transfer energy so that one $\mathrm{Eu}^{3+}$ ion is raised to an upper f-level while the other drops directly to the ${ }^{5} \mathrm{D}_{0}$ state, by-passing the ${ }^{5} \mathrm{D}_{2}$ and ${ }^{5} \mathrm{D}_{1}$ states and populating the ${ }^{5} \mathrm{D}_{0}$ state faster than it would be populated by sequential relaxation. This mechanism could be favored over sequential relaxation through the ${ }^{5} \mathrm{D}_{2}$ and ${ }^{5} \mathrm{D}_{1}$ states because the high density of states (close packing) of the upper f-levels allows energy transfer with a smaller energy mismatch (which must be dissipated by phonons) than occurs with sequential relaxation. In terms of $\mathrm{Eu}^{3+}$ emission efficiency, this interaction mechanism is neutral. Initially there are two activators in excited states with the potential for the emission of two red photons. After the interaction the two $\mathrm{Eu}^{3+}$ ions still have the potential to emit two red photons. This interaction explains the fast rise in the persistence curve (data) of Figure 4.

While the interaction shown in Figure 4 explains deviations from the sequential relaxation model, it does not account for the degradation of phosphor efficiency with increasing activator concentration. However, other types of excited activator interactions, one of which is depicted in Figure 6, have the net effect of converting potential photons into phonon (heat) energy.

A mechanism (Figure 6) for the complete de-excitation of an excited $\mathrm{Eu}^{3+}$ involves two nearby activators excited to the ${ }^{5} \mathrm{D}_{3}$ (or other ${ }^{5} \mathrm{D}$ ) state. One ${ }^{5} \mathrm{D}_{3}$ state relaxes directly to the ground state by transferring energy to promote the other excited $\mathrm{Eu}^{3+}$ to an upper level. The $\mathrm{Eu}^{3+}$ which receives the excitation relaxes nonradiatively to an emitting level. Where, potentially, two photons could be emitted, a maximum of one photon can now be emitted. The energy that could have resulted in an additional photon was dissipated nonradiatively as phonons (heat). The remaining excited $\mathrm{Eu}^{3+}$ may interact with another excited activator in the same way, essentially 
acting as a catalyst for nonradiative de-excitation. Such repeated interactions will seriously degrade phosphor efficiency.

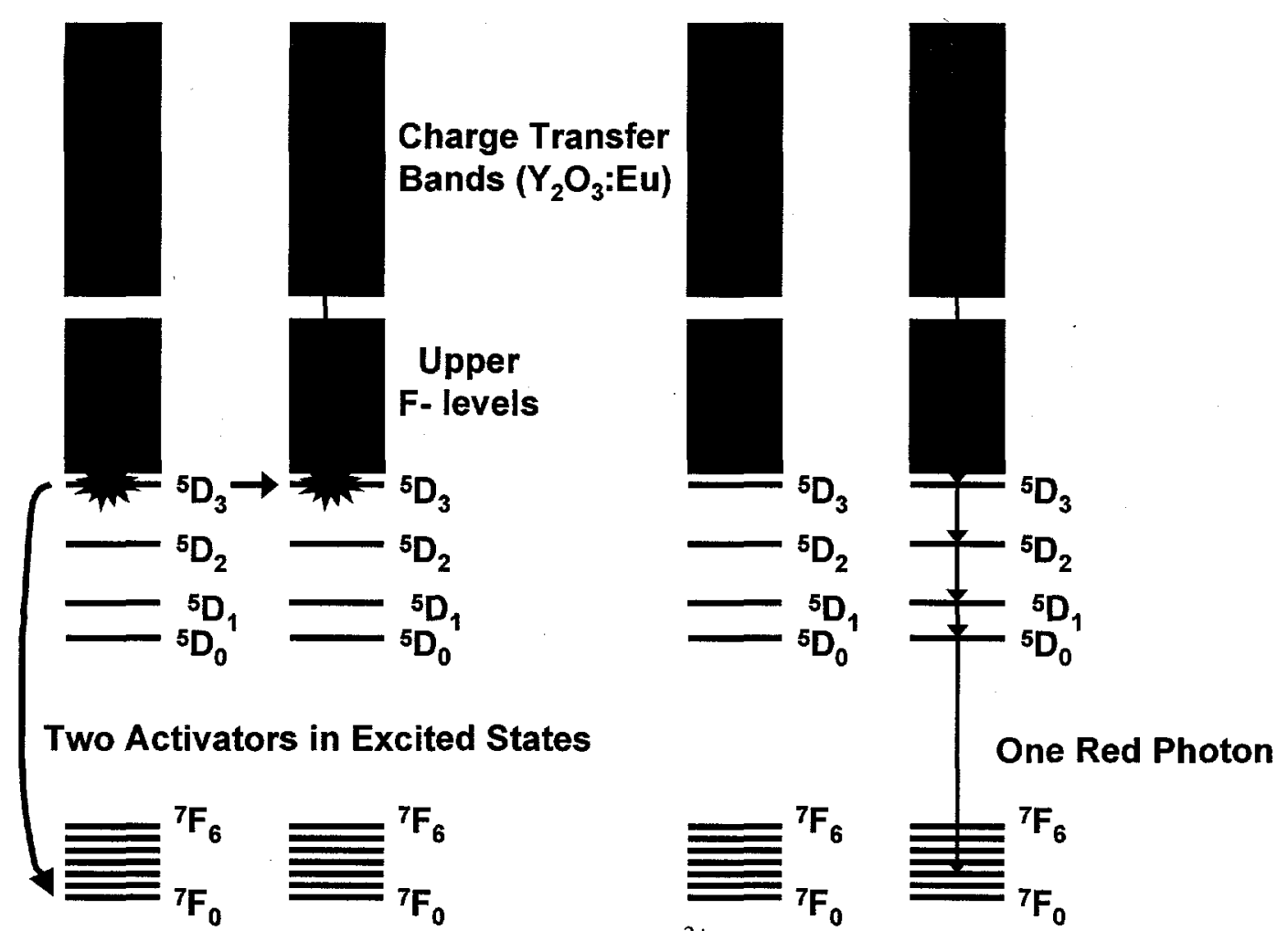

Figure 6. Complete de-excitation of a $\mathrm{Eu}^{3+}$ ion by excited state energy transfer

The two $\mathrm{Eu}^{3+}$ sites which interact as shown in Figure 6 form a type of "trap" - but not the usual concept of a trap, which involves de-excitation of a single excited activator, with the energy going to some other type of site. This type of trap requires two excited $\mathrm{Eu}^{3+}$ ions in close proximity. Individually, each $\mathrm{Eu}^{3+}$ in the "trap site" can act like a normal emitter.

One consequence of the excited state interaction mechanism is the introduction of a term involving second order kinetics (because the interaction depends on the concentrations of two activators in excited states) into the rate equations governing excited state relaxation.

Experimentally, the addition of second order rate terms should result in nonlinear semi-log persistence curves and a dependence of the shape of the persistence curves on excitation intensity. We found, at most, a very weak dependence of the persistence curve shape on excitation intensity for intermediate activator concentrations in the $\mathrm{Y}_{2} \mathrm{O}_{3}: \mathrm{Eu}$ and $\mathrm{Y}_{2} \mathrm{SiO}_{5}: \mathrm{Tb}$ systems. Future experiments wll measure the effect of excitation intensity on the persistence curves of powders with higher activator concentrations.

Transition metal activators might be expected to show enhanced excited state interactions compared to rare earth activators, since the valence-band d-orbitals of transition metals extend further from the activator ion than the f-orbitals of rare earths. Activators with d-orbitals should be able to interact with other (especially excited state) activators over longer distances. The shape of the persistence curve of $\mathrm{Zn}_{2} \mathrm{SiO}_{4}$ with 10 atomic \% $\mathrm{Mn}$ was found to show a strong dependence on excitation intensity, as shown in Figure 7. 


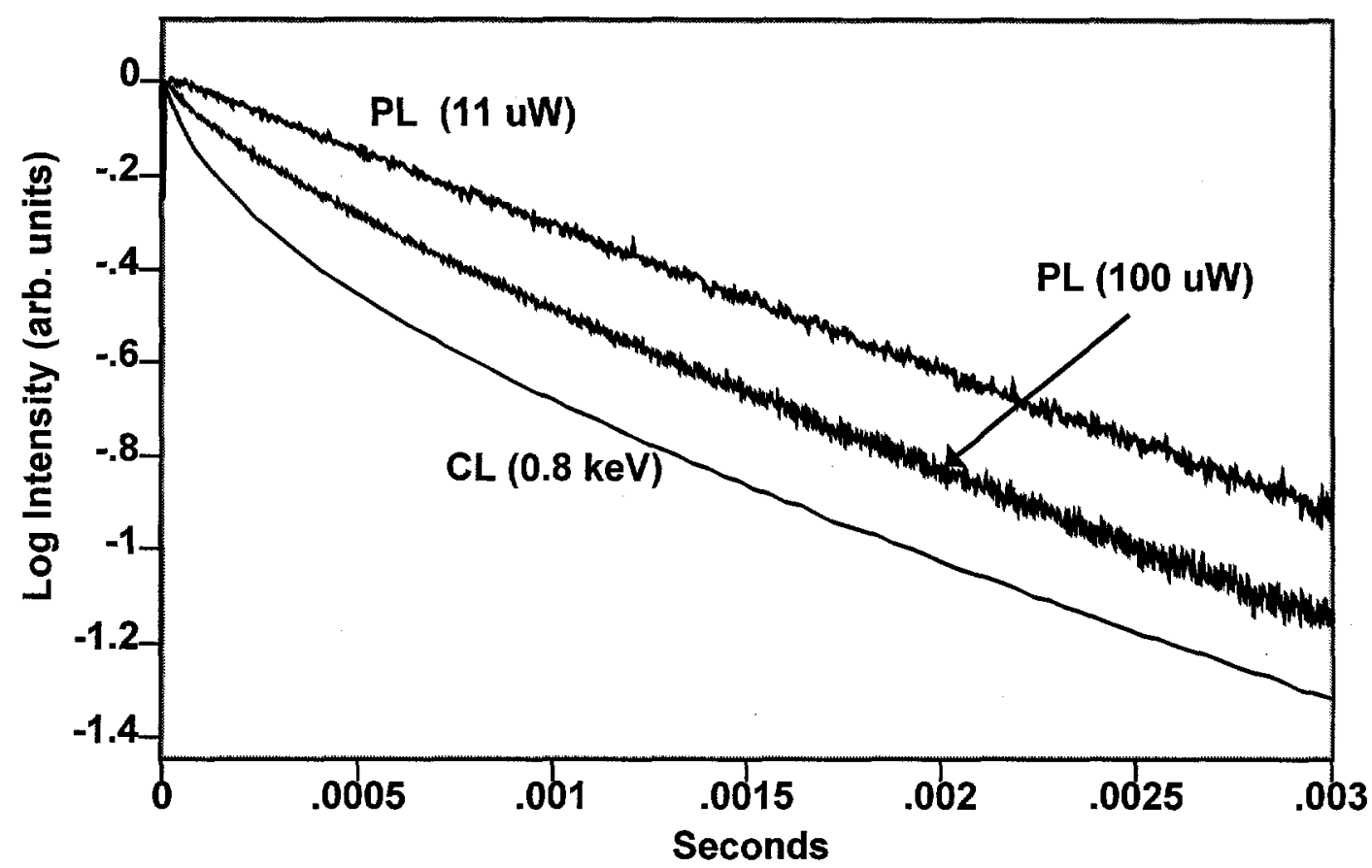

Figure 7. Semi-log plot of persistence from $\mathrm{Zn}_{2} \mathrm{SiO}_{4}$ with 10 atomic $\% \mathrm{Mn}$, monitoring green emission $(530 \mathrm{~nm})$. PL was excited at $232 \mathrm{~nm}$ with laser intensities of $11 \mathrm{uW}$ and $100 \mathrm{uW}$. CL was excited at $0.8 \mathrm{keV}$.

The semi-log plot of persistence from $\mathrm{Zn}_{2} \mathrm{SiO}_{4}$ with 10 atomic \% Mn excited at low (PL) excitation intensity $(11 \mathrm{uW})$ is nearly linear and is consistent with first order (isolated activator) kinetics. The long tail of the persistence resulting from higher (PL) excitation intensity $(100 \mathrm{uW})$ is also linear and has the same slope (lifetime) as the persistence resulting from lower excitation intensity. At shorter times the persistence resulting from higher excitation intensity is distinctly nonlinear. The nonlinear portion of this curve is modeled well if terms second-order in excited activator concentration (corresponding to excited activator interactions) are added to the rate equation for excited state relaxation. It is expected that a higher density of excited activators, resulting from higher excitation intensities, results in enhanced excited state interactions. The changes in the persistence curves due to nearly an order of magnitude difference in (PL) excitation intensity can be replicated by only a $20 \%$ change in manganese concentration in the host $\mathrm{Zn}_{2} \mathrm{SiO}_{4}$. At least for the excitation intensities used in PL excitation, the concentration at which activator is doped into the host phosphor matrix appears to have a stronger effect on the rate of excited state interactions than the excitation intensity. Increased activator concentration will both reduce the average distance between activators in the host matrix and increase the absorption of photons which cause excitation.

The semi-log plot of the persistence curve from $\mathrm{Zn}_{2} \mathrm{SiO}_{4}$ with 10 atomic $\% \mathrm{Mn}$ obtained by electron beam excitation (Figure7, CL with $0.8 \mathrm{keV}$ electrons) shows significantly more curvature, implying even higher rates of excited state interaction than was achieved for PL at the highest excitation intensity. The persistence curves of $\mathrm{Zn}_{2} \mathrm{SiO}_{4}$ with 10 atomic $\% \mathrm{Mn}$ obtained with electron energies greater than $0.8 \mathrm{keV}$ have less curvature and lie between the $(0.8 \mathrm{keV}) \mathrm{CL}$ 
persistence curve and the (100 uW) PL persistence curve. CL apparently achieves higher local densities of excited activators than PL in these experiments.

\section{CONCLUSIONS}

We have presented evidence for the existence of interactions between excited state activators. Certain forms of these interactions explain why persistence curves deviate from the relaxation model, which assumes that excitation relaxes sequentially between excited states of an activator. Other forms of these interactions completely de-excite activators and can seriously degrade phosphor efficiency by converting energy which potentially can be emitted as photons into lattice phonons. The efficiency-degrading interactions explain the decrease in phosphor efficiency as activator concentration increases

Three factors potentially influence the rate at which these excited state interactions occur with excitation by photons (PL):

1. Activator concentration - determines the average distance between activators and, hence, the rate of excited state interactions and also affects the amount of excitation energy absorbed; 2. Resonant energy transfer - effects migration of excitation to activators in close proximity, i.e., where they can interact; and

3. Excitation intensity - determines the initial concentration of excited activators.

Throughout this paper we have presented data obtained by electron beam excitation (CL) comparison to that obtained by photon absorption (PL). The trends related to excited state interactions are uniformly exaggerated in the CL data compared to the PL data. We believe that a primary difference between CL and PL excitation is a much greater local density of excited activators along the track of an impinging electron than is achieved in the phosphor matrix by the (photon) excitation intensities used in our PL experiments. It is this high local density of excited activators which enhances excited state interactions in CL experiments.

Excited state interactions appear to intrinsically limit phosphor efficiency. However, there may be ways to ameliorate their effects. First, preparation techniques which minimize aggregation of activators into locally high concentrations in the phosphor matrix will minimize excited state interactions. Phosphors prepared by spray pyrolysis achieve higher efficiencies with low energy CL excitation than those prepared by conventional techniques. This improvement in efficiency may be due to less local aggregation of activators when spray pyrolysis is used to synthesize the phosphors. Second, there may be dopants, which, when inserted into the phosphor matrix, block interactions between excited state activators. Miller [8] has found that rare earth dopants with energy states near resonant with those of $\mathrm{Eu}^{3+}$ in $\mathrm{Y}_{2} \mathrm{O}_{3}$ : Eu tend to quench its emission, but dopants with no resonant energy states may enhance its emission. We plan to investigate these means of enhancing phosphor efficiency.

\section{ACKNOWLEDGMENTS}

Funding was provided by DARPA and the U.S. DOE. The authors thank Superior MicroPowders for providing the phosphor materials used in this study. Sandia National Laboratories is a multiprogram laboratory operated by Sandia Corporation, a Lockheed Martin Company, for the United States Department of Energy under Contract DE-AC04-94AL85000. 


\section{REFERENCES}

1. L. Ozawa, J. Electrochem. Soc. 126(1), 106-109 (1979).

2. U. Vater, G. Kunzler and W. Tews, Journal of Fluorescence 4, 79-82 (1994).

3. C. H. Seager and D. R. Tallant, "The Role of Activator-Activator Interactions in Reducing Low-Voltage-Cathodoluminescence Efficiency in Eu and Tb doped Phosphors," to be published in J. Appl. Physics.

4. J. Heber and U. Kobler, Izv. Akad. Nauk SSSR, Ser. F., 37(4), 772-777 (1973)

5. J. Heber, K. H. Hellwege, U. Kobler and H. Murmann, Z. Physik 237, 189-204 (1970).

6. R. B. Hunt Jr. and R. G. Pappalardo, Journal of Luminescence, 34, 133-146 (1985).

7. D. R.Tallant, M. P. Miller and J. C. Wright J. Chem. Phys. 65(2), 510-521 (1976).

8. M. J. Fuller, J. Electrochem. Soc., 128(6), 1381-3 (1981). 